\title{
LA GENERALIZACION DE LA PRODUCCION DE MERCANCIAS Y LA MODERNIZACION PRODUCTIVA DE LA AGRICULTURA EN GALICIA, 1876-1976
}

\author{
MANUEL JAIME BARREIRO GIL \\ Universidad de Santiago
}

El proceso desamortizador permitió una reestructuración capitalista del campo español al remover todo el sistema de relaciones de producción propias del Antiguo Régimen en favor de la propiedad burguesa de la tierra. Era un paso necesario para que el modo de producción capitalista pudiese desarrollarse con plenitud en España ${ }^{1}$.

Por otra parte, la apertura del proceso de crecimiento económico moderno que alcanza a la sociedad española de finales del siglo $\mathrm{XIX}^{2}$ exige la elevación del nivel de mercantilización de la actividad económica o, si se prefiere, la generalización de la práctica de producir para intercambiar. También la desamortización permitió la aceleración de este proceso en tanto que, por el volumen de tierras puestas en circulación, afectó sustancialmente a las condiciones imperantes para el cálculo económico, es decir, para la organización de la producción.

Las exigencias mínimas del proceso de cambio eran fundamentalmente dos: en primer lugar, el establecimiento de una separación cada vez más drástica entre las diferentes actividades productivas, y en segundo, la conversión en mercancías de todos los factores de la producción.

Sin embargo, también es sabido que el proceso de unificación que implica la formación de un mercado nacional es desigual en el tiempo y en el espacio. Su nivel de implicaciones económicas, políticas, culturales, lingüísticas, geofísicas e incluso sociológicas en la constitución de un nuevo «bloque histórico» que lo sustancia ${ }^{3}$ es muy elevado y amplio, lo cual impide cualquier tipo de linealidad.

' J. Fontana, "Transformaciones agrarias y crecimiento económico en la España contemporánea", en Cambio económico y actitudes politicas en la España del siglo XIX, Barcelona, 1975, pp. 147-212.

2 J. NADal, El fracaso de la revolución industrial en España, 1814-1913, Barcelona, 1977.

${ }^{3}$ E. Sereñ, Capitalismo y mercado nacional, Barcelona, 1880. 


\section{Galicia: la persistencia de las estructuras tradicionales}

Esa etapa de cambio es, en Galicia, más dilatada que en otras zonas del área peninsular. La persistencia del contrato foral como marco general de regulación de la propiedad y posesión de la tierra ${ }^{4}$ anuló cualquier logro del esfuerzo por convertir a la tierra en mercancía. En Galicia, los que se adjudicaban la subasta de los bienes desamortizados no adquirían la propiedad de la tierra, sino únicamente el derecho a reclamar una renta anual de un usufructuario que la cultivaba, en la mayor parte de las ocasiones desde hace un largo período de tiempo y que ahora difícilmente podía ser expulsado de ella ${ }^{5}$.

En Galicia el proceso desamortizador no supuso un cambio sustancial de las estructuras agrarias; menos todavía una reestructuración capitalista del agro. Al contrario, una vez concluido persisten incólumes las formas propias del antiguo régimen económico en el foro, sin que se haya constituido un espacio significativo en el que las reglas sean la propiedad burguesa de la tierra y el cálculo económico de tipo capitalista para la actividad productiva.

Por estas razones, los procesos productivos agrarios que se llevan a cabo en Galicia operan con una cierta «autonomía» respecto de lo que sucede con la agricultura y la economía españolas. Se establece aquí una distancia que no será recuperada cuando, ya en la década de los años 1960, la producción agraria gallega se integra de manera funcional y subordinada en la operatividad del modelo general de industrialización de España.

\section{Mercados y mercancias en la Galicia del siglo XIX}

De todo lo dicho no podemos derivar la conclusión de que los productores agrarios gallegos no hayan participado en la práctica de la producción de mercancías hasta la segunda mitad del siglo xx. Es cierto que hasta ese momento existían amplios espacios en los que prevalecía el sistema de «policultivo de subsistencia» ${ }^{6}$, con una relación equilibrada entre la actividad agraria y la artesanía rural y ciertas prácticas de intercambio no monetario, fundamentalmente en el ámbito de la circulación de la fuerza de trabajo. Pero no por ello podemos hablar, sin matices, de una «economía cerrada» para caracterizar la agricultura gallega de finales del siglo xIX y principios del $\mathbf{x x}$.

4 M. J. BARreiro GII, "Aportación a unha bibliografía sobor da cuestión foral galega", en Revista Galega de Estudios Agrarios, núm. 2, 1979, pp. 327-357.

5 A. Palomar, "Foros", en Nueva Enciclopedia Jurídica, Barcelona, 1976, t. X, páginas 98-133.

- J. Garcta Fernández, Organización del espacio y economía rural en la España atlántica, Madrid, 1975; A. BOUнIER, La Galice. Essai geographique d'analyse et d'interpretation d'un vieux complexe agraire, Poitiers, 1979. 
Un cierto grado de autarquía comarcal y, como consecuencia, una escasa integración económica en la España del Antiguo Régimen es un hecho constatado. «En lo que se refiere al reino de Galicia (...) se puede deducir que la precariedad de las comunicaciones era un hecho en casi toda su geografía (...) y el campesinado vivía al límite de la subsistencia (...) ${ }^{7}$, síntoma y causa, respectivamente, de la escasa demanda de tráficos comerciales y la no existencia de un comercio organizado. Grado de autarquía que se pone de manifiesto, asimismo, en el excesivo número de ferias y mercados que con dimensión tan sólo comarcal, cuando no simplemente local, se celebran anualmente en Galicia (más de 4.500); la mayor parte de ellos, como puede comprenderse, de muy escasa entidad, tanto por el volumen de las transacciones que en ellos se realizan como por lo reducido de sus respectivas áreas de influencia. La escasa circulación de mercancías tiene un índice, que es generalmente aceptado, en las diferencias existentes entre unas zonas y otras del país en cuanto al mecanismo de formación de los precios. También en las bajas cantidades percibidas como derechos sobre el tránsito por los titulares de barcajes, peajes, portazgos o similares. El análisis de las estadísticas de los precios y de la producción de los principales productos agrarios muestra la inexistencia de un mercado transparente y articulado. Durante el siglo xIx la agricultura de Galicia continúa subordinada a los cultivos y técnicas tradicionales. Prevaleció el policultivo minifundista, y sobre él, el sistema de cargas que articulaba el contrato foral. Hasta muy a finales de siglo el sistema general de comunicaciones y medios de transporte era insuficiente e inadecuado y estaba, además, afectado por un crónico mal estado. Las crisis agrarias de subsistencia tienen efectos devastadores sobre los habitantes del país. Galicia está presa en las redes del atraso económico y su población tan sólo es capaz de luchar, con escasos éxitos, por la eliminación de la miseria en sus condiciones de vida cotidiana ${ }^{8}$.

\section{Especialización productiva, modernización agraria y mercantilización de la producción, 1880-1930.}

Si «antes de 1880 parece ser que hay que tratar de dos precios, el de las provincias maríticas y el de las interiores», a partir de ahí los precios de

7 J. Garcha-Lombardero, La agricultura y el estancamiento económico de Galicia en la España del Antiguo Régimen, Madrid, 1973, pp. 63-65.

8. Garcia-Lombardero y M. J. Barreiro Gir, "La articulación del mercado gallego y la estructura de las comunicaciones en su perspectiva histórica", ponencia presentada al coloquio de Pau de 1981. 
las cuatro provincias se concentran en torno a un precio regional y parece que empieza a formarse lo que se podría denominar un «mercado regional» ${ }^{9}$.

Entre las causas que han promovido este proceso de articulación social que encarna la del mercado podemos encontrar, entre otras: el aumento de las importaciones de granos, que contribuyen a atenuar las crisis y a unificar los precios; el mayor volumen de realización de las obras de construcción y mejora de las líneas férreas y caminos, y los efectos que, a través del comercio exterior, tiene sobre la agricultura y economía gallegas en general el proceso de crecimiento económico que están conociendo los países europeos, especialmente Inglaterra y, en menor grado y más tarde, la propia España.

\subsection{LA CRISIS DEFINITIVA DEL SISTEMA FORAL}

Durante la segunda mitad del siglo XIX se producen abundantes manifestaciones que demuestran cómo se amplía progresivamente la conciencia social acerca de la conveniencia y necesidad de un profundo cambio destinado a romper las condiciones del equilibrio aparente en que se reproducen las del atraso económico. Sobre todo, en lo que aquí interesa, se acepta con igual amplitud el hecho de que para la supresión del estado ruinoso en que se encontraban la agricultura y la economía gallegas era necesario reformar en profundidad las estructuras vigentes, porque, en gran parte, ellas mismas eran las causantes del desequilibrio que se producía entre las necesidades de la población y la capacidad del sistema económico para satisfacerlas.

En el año 1864, con este espíritu, la Sociedad Económica de Amigos del País de Santiago convoca un congreso que tendría por objeto casi exclusivo tratar de «si conviene el cambiar el sistema general de constitución de la propiedad gallega», y más en concreto, «si es justo, político, económico y oportuno el suprimir el sistema foral» ${ }^{10}$. El hecho de que en la Galicia de entonces se ponga en duda lo conveniente de la vigencia de una relación contractual que afecta a la mayor parte de la superficie agraria del país, es suficiente para informar de cuán ampliamente se había extendido ya la conciencia de crisis. Tengamos presente, además, que los asistentes, «370 señores..., todos ellos de los más distinguidos en sus clases respectivas" ", no son, en su inmensa mayoría, sino perceptores de rentas forales.

\footnotetext{
- J. Garcta-Lombardero, "La formación de un mercado regional: Galicia, 18601890", en Moneda y Crédito, núm. 119, 1971, pp. 67-75

$10 \mathrm{~J}$. Planellas, Congreso agrícola gallego de 1864. Actas, discursos o sus estratos $y$ demás documentos de que se dio cuenta en esa reunión memorable, Santiago, 1864.

"Ibidem.
} 
Es cierto que el congreso no dio respuesta a aquella cuestión crucial ${ }^{12}$. Los participantes no tenían un programa para el crecimiento económico que supusiera una alternativa global al contrato foral y a la propia organización de la producción agraria. Sin embargo, su reunión había servido para firmar el acta de derrumbe del viejo aparato social del Antiguo Régimen, todavía plenamente vigente en Galicia; sus protagonistas habían perdido la confianza en él.

En el país se estaba produciendo un triple proceso de especialización productiva, modernización de la agricultura y mercantilización de la producción agraria que incluso en sus manifestaciones cotidianas empezaba a encontrar estrechos los márgenes funcionales que le concedía el caduco sistema foral.

\subsection{La COMERCIALIZACIÓN DE LA PRODUCCIÓN AGRARIA}

Desde la segunda mitad del siglo xvini se constata la importancia que en Galicia tiene la producción de ganado vacuno, tanto como auxiliar de la mano de obra agrícola y abastecedor de alimentos, como en su funcionalidad como valor de cambio comercializable. Función esta última particularmente remarcable cuando, a partir de la apertura del mercado inglés para los bueyes cebones gallegos a mediados del siglo xix, el ganado va a ser el principal y casi único producto de exportación al extranjero y, por tanto, el principal y casi único producto capaz de obtener en el mercado los recursos financieros necesarios para la explotación agraria ${ }^{13}$.

Entre 1868 y 1886 Galicia exportaba un promedio anual de casi 36.000 cabezas de ganado vacuno, volumen comercial de envergadura, al menos en tanto que el promedio español en todo su conjunto tan sólo lo superaba en algo más de 10.000 reses anuales. Y cuando se cierra el mercado de exportación a Inglaterra por causa de las nuevas normas de competencia que se exigen al producto gallego ${ }^{14}$, Galicia no tarda en lograr un nuevo ámbito de

${ }^{12}$ En su sesión del día 26 de julio, el congreso aprobaba una proposición presentada por el sector más conservador que rezaba de la siguiente manera: "En atención a la considerable importancia del contrato foral en Galicia, a la honda transformación que su reforma improvisada produciría (...), que se aplace la resolución definitiva del tema (...) para otro congreso agrícola." J. Planellas, op. cit., página 98.

${ }_{13}$ En esta cuestión seguimos a X. Carmona Badfa, "Sobre as orixens da orientación exportadora da producción bovina galega. As exportacións a Inglaterra na segunda mitade do século xIX", en Grial, Extra Historia, 1982; J. García-LombarDERO, "Evidencias dunha crise agraria en Galicia: precios e exportación de gando a remates do século xIx", en Revista Galega de Estudios Agrarios, núm. 1, 1979, páginas 53-68; R. VILLARES PAz, "Transformaciones técnicas da agricultura e comercialización do gando bovino. Galicia, 1900-1936", en Cuadernos de Estudios Gallegos, t. XXXI, 1978-1980, pp. 209-243.

i4 J. Garcí-Lombardero, "Evidencias...". 
comercialización de carne de vacuno, todavía más amplio, abasteciendo a la propia España. Entre 1907 y 1931 en el conjunto de estaciones de ferrocarril establecidas por la Compañía de El Norte entre Quereño y La Coruña se facturaron, con destino al interior de España, 3.771 .527 reses, lo que supone una media anual de 150.340 cabezas de vacuno ${ }^{15}$. Entre los años 1889 y 1928 la red de Galicia de la Compañía ferroviaria M.Z.O.V. transportó 1.973 .751 reses, equivalentes a un promedio de 49.343 anuales ${ }^{16}$. La evolución de los promedios anuales de comercialización de ganado vacuno experimenta un

Volumen de comercialización de ganado vacuno en Galicia, 1842-1931 (Promedios anuales por decenios)

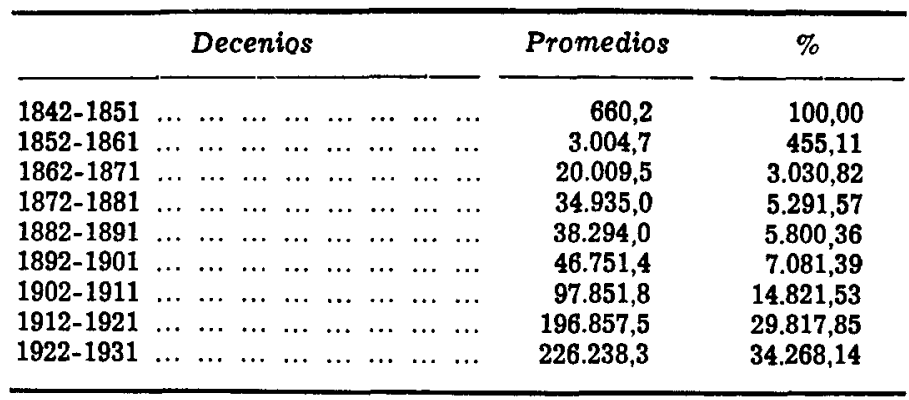

FunNte: Véase nota número ${ }^{17}$.

is Ministerio de Agricultura, Industria y Comercio. Dirección General de Ganadería e Industrias Pecuarias, Estudio del peso en vivo, rendimientos, precios y cotizaciones del ganado bovino de abasto de Galicia y Asturias, con expresión de las reses vacunas de Galicia facturadas por ferrocarril con destino al interior de España durante veinticinco años, Madrid, 1932.

${ }^{16}$ A partir de las memorias anuales de la Compañía.

17 Las cifras base para realizar los cálculos que se recogen en el cuadro proceden de la suma de las cabezas de ganado vacuno exportadas a Inglaterra y Portugal, tomadas de $\mathrm{X}$. CARMona, art. cit.; las cabezas de ganado vacuno de Galicia embarcadas en las estaciones ferroviarias situadas entre Quereño y La Coruña con destino al interior de España, tomadas del Ministerio de Agricultura, INDustria y Comercio. Dirección General de Ganaderia e Industrias Pecuarias, op. cit., y, por fin, el número de reses de vacuno transportadas por la línea de Galicia de la Cía. M.Z.O.V., obtenidas en las propias memorias anuales de la misma. Debe tenerse en cuenta que estas cifras no son más que las que contienen las únicas estadísticas sistemáticas disponibles sobre el tema y no se corresponden a la totalidad del ganado vacuno comercializado en Galicia. No se dispone de ninguna información acerca de lo que sucede en cada una o alguna de las ferias o mercados ganaderos que se celebran en el país, ni sistemática ni asistemáticamente. Tampoco ha llegado a nosotros ningún tipo de información acerca del comportamiento de los "tratantes", intermediarios casi omnipresentes en las transacciones de ganado. Nada sabemos, tampoco, del posible o incierto transporte de ganado por carretera o que se dirige a países extranjeros sin cumplir los requisitos aduaneros pertinentes. 
ascenso que los lleva de las 660 reses durante el decenio de 1842.1851 a las 226.238 durante el que va desde 1922 a 1931 .

Esta evolución, tan espectacularmente positiva, del volumen de ganado vacuno comercializado es comprensible. La coyuntura del mercado era favorable para el producto cárnico de vacuno procedente de Galicia; hasta la comercialización en masa de las producciones cárnicas de Argentina y Nueva Zelanda, el producto gallego gozaba de una posición de casi monopolio de oferta en el mercado inglés, al igual que la que disfrutaría luego en el abastecimiento de carne de ternera a Madrid y Barcelona. Disponemos de información acerca de la evolución de los índices de precios en España a partir de 1913, en los que se evidencia suficientemente lo que se dice.

Indices de precios en España, 1913-1930*

\begin{tabular}{|c|c|c|c|c|c|c|c|c|}
\hline & & (1) & (2) & (3) & (4) & (5) & (6) & (7) \\
\hline 1913 & $\begin{array}{lllll}\ldots & \ldots & \ldots & \ldots & \ldots\end{array}$ & 100,0 & 100,0 & 100,0 & 100,0 & 100,0 & 100,0 & 100,0 \\
\hline 1914 & $\ldots$ & 94,3 & 100,6 & 127,3 & 100,0 & 1000 & 98,4 & 100,3 \\
\hline 1915 & $\begin{array}{llllll}\ldots & \ldots & \ldots & \ldots & \ldots\end{array}$ & 106,8 & 94,7 & 227,3 & 120,0 & 108,1 & 118,3 & 107,9 \\
\hline 1916 & $\begin{array}{llllll}\ldots & \ldots & \ldots & \ldots & \ldots\end{array}$ & 117,1 & 138,2 & 218,2 & 169,7 & 140,5 & 141,0 & 120,0 \\
\hline 1917 & $\begin{array}{cccccc}\ldots & \ldots & \ldots & \ldots & \ldots \\
\ldots & \ldots & \ldots & \ldots & \ldots\end{array}$ & 129,0 & 143,4 & 172,7 & 205,7 & 189,2 & 165,6 & 147,8 \\
\hline 1918 & $\begin{array}{llllll}\ldots & \ldots & \ldots & \ldots & \ldots\end{array}$ & 153,8 & 185,7 & 218,2 & 308,6 & 270,3 & 204.9 & 166,1 \\
\hline 1919 & $\begin{array}{llllll}\ldots & \ldots & \ldots & \ldots & \ldots\end{array}$ & 160,9 & 192,0 & 281,8 & 285,7 & 297,3 & 204,2 & 203,4 \\
\hline 1920 & $\begin{array}{llllll}\ldots & \ldots & \ldots & \ldots & \ldots\end{array}$ & 202,7 & 207,3 & 289,4 & 285,7 & 297,3 & 223,4 & 203,6 \\
\hline 1921 & $\begin{array}{llllll}\ldots & \ldots & \ldots & \ldots & \ldots\end{array}$ & 163,4 & 175,6 & 233,3 & 211,4 & 283,8 & 184,5 & 189,4 \\
\hline 1922 & $\begin{array}{lllll}\ldots & \ldots & \ldots & \ldots & \ldots\end{array}$ & 137,9 & 141,7 & 281,4 & 151,9 & 226,3 & 172,5 & 208,7 \\
\hline 1923 & $\ldots$ & 134,2 & 140.7 & 221,2 & 1429 & 244.4 & 170,9 & 200,0 \\
\hline 1924 & $\begin{array}{llllll}\ldots & \ldots & \ldots & \ldots & \ldots\end{array}$ & 135,7 & 158,7 & 287,1 & 147,2 & 242,5 & 181,9 & 210,3 \\
\hline 1925 & $\begin{array}{llllll}\ldots & \ldots & \ldots & \ldots & \ldots\end{array}$ & 158,5 & 175,8 & 299,2 & 1468 & 208,1 & 185,0 & 225,1 \\
\hline 1926 & $\begin{array}{ccccc}\ldots & \ldots & \ldots & \ldots & \ldots \\
\ldots & \ldots & \ldots & \ldots\end{array}$ & 151,2 & 149,9 & 231,8 & 140,5 & 202,7 & 174,8 & 237,5 \\
\hline 1927 & $\begin{array}{llllll}\ldots & \ldots & \ldots & \ldots & \ldots & \ldots\end{array}$ & 154,9 & 158,7 & 272,0 & 137,1 & 189,2 & 167,9 & 221,7 \\
\hline 1928 & $\begin{array}{lllll}\ldots & \ldots & \ldots & \ldots & \ldots\end{array}$ & 157,6 & 164,6 & 219,7 & 123,3 & 202,7 & 162,6 & 194,9 \\
\hline 1929 & $\begin{array}{ccccc}\cdots & \cdots & \cdots & \cdots & \ldots \\
\ldots & \ldots & \ldots & \ldots\end{array}$ & 156,8 & 167,3 & 237,1 & 1278 & 2027 & 167,7 & 203,7 \\
\hline 1930 & $\begin{array}{llllll}\ldots & \ldots & \ldots & \ldots & \ldots\end{array}$ & 147,9 & 167,6 & 213,6 & 137,7 & 204,3 & 167,0 & 214,8 \\
\hline
\end{tabular}

* Hasta 1919 se trata de índices simples, y a partir de ahf ponderados.

(1) Trigo; (2) Maíz; (3) Patata; (4) Superfosfatos de cal; (5) Madera de pino rojo; (6) General; (7) Carne de ternera de Galicia.

Fuente: E. Paris Eguilaz, El movimiento de los precios en España, Madrid, 1943 , pp. 17-68.

La observación de las últimas columnas del cuadro precedente, que corresponden a la evolución del índice general de precios y al de la carne de tercera en Galicia, muestra claramente la situación de ventaja que, durante este período, pudo ocupar el producto galaico en el conjunto del mercado 
español. No es de extrañar que esto haya servido de incentivo para una atención preferente a la producción ganadera en este subsector. La Asociación General de Ganaderos explicaba este fenómeno considerando que, como

«efecto del renacimiento económico de España desde 1914 a la fecha, se ha difundido el bienestar, se han modificado las costumbre y el país, menos interesado por el ahorro y por el sacrificio, se ha decidido por un mayor consumo en todos los órdenes (...), el consumo de carnes ha sido el más afectado en este aspecto, [añadiendo que] la demanda española prefiere reses jóvenes por aversión a la grasa (...); Barcelona sólo consume ternera de menos de dos años de edad (...); Galicia es una gran productora con altos excedentes, que abastece a Madrid y Barcelona» ${ }^{18}$.

\subsection{Cambios en el sistema de cultivos}

El productor rural gallego no hace sino ceñirse lo más escrupulosamente que le permiten hacerlo sus condiciones de producción a las directrices emanadas del comportamiento general del mercado peninsular. Es evidente que un volumen de comercialización tan elevado de reses de vacuno exige, aunque sea primariamente, una cierta especialización de la producción dirigida precisamente a mejorar las condiciones de cría y engorde de este tipo de ganado. Es por ello comprensible que se produzcan modificaciones sustanciales en el sistema de cultivos a favor de los pratenses y forrajeros y a costa de otros tradicionales más propios del policultivo minifundista de subsistencia. Esta dinámica es similar a la que se experimenta en la Europa del último tercio del siglo $\mathrm{xIX}$ y primero del $\mathrm{xx}$, con programas fomentados $\mathrm{y}$ difundidos por todo tipo de instituciones políticas, profesionales o culturales. Todo ello en un proceso de cambio que abre la crisis de la agricultura tradicional en Galicia, puesta de manifiesto en el aumento de las actividades puramente comerciales, la expulsión acelerada de mano de obra del campo ${ }^{19}$, la progresiva separación de las actividades productivas que se traduce en la desaparición casi total de la artesanía rural y la intensificación del uso del suelo cultivable ${ }^{20}$.

\footnotetext{
18 Asociación General de Ganaderos, Producción y consumo de carne y productos lácteos en España, Madrid, 1927.

19 X. A. López Taboada, Poboación e economía en Galicia, A Coruña, 1979; J. M. Beiras TorRado, Estructuras y problemas de la población gallega, La Coruña, 1970.

20 X. M. Bemas Torrado, O atraso económico de Galicia, Vigo, 1972.
} 


\section{Superficies ocupadas por los distintos grupos de cultivos} (Hectáreas estimadas)

\begin{tabular}{|c|c|c|c|}
\hline & $1903-1912$ & 1923 & $\%$ \\
\hline $\begin{array}{llllllllll}\text { Cereales } & \ldots & \ldots & \ldots & \ldots & \ldots & \ldots & \ldots & \ldots & \ldots \\
\text { Leguminosas } & \ldots & \ldots & \ldots & \ldots & \ldots & \ldots & \ldots & \ldots \\
\text { Lortícolas } & \ldots & \ldots & \ldots & \ldots & \ldots & \ldots & \ldots & \ldots & \ldots \\
\text { Industriales } & \ldots & \ldots & \ldots & \ldots & \ldots & \ldots & \ldots & \ldots \\
\text { Indula } & \ldots & \ldots & \ldots & \ldots & \ldots & \ldots \\
\text { Pecuarios } & \ldots & \ldots & \ldots & \ldots & \ldots & \ldots & \ldots & \ldots & \ldots\end{array}$ & $\begin{array}{r}448.024 \\
145.800 \\
11.321 \\
1.171 \\
1.177 .741\end{array}$ & $\begin{array}{r}321.575 \\
107.145 \\
10.644 \\
1.327 \\
1.517 .149\end{array}$ & $\begin{array}{r}-28,32 \\
-26,51 \\
-\quad 5,98 \\
+\quad 13,32 \\
28,81\end{array}$ \\
\hline
\end{tabular}

Futente: Véase nota número ".

El cuadro que antecede es preciso leerlo con prudencia, primero porque lo exige la muy limitada exactitud de las fuentes españolas de estadísticas agrarias; segundo, porque algunos de los cambios tienen significado biunívoco, como por ejemplo la pérdida de espacio por parte de los cereales, que es más que compensada por la intensificación del cultivo del maíz, así como también la pérdida de los productos hortícolas vino motivada en parte por la expansión del cultivo de la patata; en tercer lugar, los aprovechamientos forrajeros no sólo proceden de lo que hemos calificado como grupo de los cultivos pecuarios, puesto que las pajas de los cereales, especialmente del maíz, son usadas con gran amplitud en la alimentación del ganado; por último, ha de tenerse en cuenta que en Galicia los cultivos de los principales cereales y los de las principales leguminosas son cultivos asociados que se obtienen sobre una misma superficie. Con estas observaciones, que no son las únicas posibles, y ateniéndome al valor estadístico de las fuentes, he realizado un cálculo que puede valorarse como razonable. Su resultado, en todo caso, no desdice de lo señalado por el profesor A. Flores de Lemus para el conjunto de la economía española ${ }^{22}$.

${ }^{21}$ El cuadro está elaborado a partir de Ministerio de Fomento. Dirección General de Agricultura, Minas y Montes. Junta Consultiva Agronómica, Avance estadístico de la riqueza que en España representa la producción media anual en el decenio 1903-1912 de cereales, leguminosas, vid $y$ olivo $y$ aprovechamientos diversos derivados de estos cultivos. Resumen hecho por la Junta Consultiva Agronómica de las memorias de 1913, remitidas por los ingenieros del Servicio Agronómico Provincial, Madrid, 1915, y Avance estadistico de la riqueza que en España representa la producción media anual en 1912 de prados, pastos y algunos aprovechamientos $y$ pequeñas industrias zoogenas anexas, Madrid, 1914, y Avance estadístico de la producción agrícola de España de 1923, Madrid, 1925; M. A. Aldso, A. Martinez y X. RAmos, A agricultura galega dos anos 1900 a 1930 ao traveso da análisis da superficie $e$ producción agrarias (trabajo de curso inédito, realizado por los autores en el Departamento de Historia Económica de la Facultad de Ciencias Económicas de Santiago, 1981); M. J. Barreiro Gil, Población, propiedad de la tierra y formación del mercado agrario en Galicia, 1900-1930, Santiago (tesis doctoral), 1983.

22 A. FlORES DE LEMús, "Sobre una dirección fundamental de la producción rural española", escrito en 1925 y reproducido en Hacienda Pública Española, números 42-43, 1976, pp. 471-485. 
La variación que experimentan las hectáreas ocupadas por los cultivos pecuarios es, como se ve, muy significativa. Desde mi punto de vista, lo sería igualmente si los resultados de los cálculos anteriores no ofreciesen más que una variación porcentual inferior en la mitad. Porque una variación limitada en el sistema de cultivos puede tener consecuencias trascendentales dentro de un sistema agrario en el que resulta fundamental el equilibrio entre los diversos cultivos. El monte, el labradío y los pastizales forman una unidad operativa en una agricultura minifundista de tipo familiar y tradicional como la gallega. Prescindir tan sólo de una parte de estos espacios exige una remodelación total de los demás y de la propia organización de la actividad productiva.

Este proceso de quiebra de las condiciones de equilibrio de la agricultura tradicional en Galicia, que alcanza un alto grado de consumación a raíz de la industrialización española de la segunda mitad del siglo $\mathrm{xx}^{23}$, ya tiene su carácter irreversible en el transcurso del primer tercio del siglo actual.

\subsection{LA UTILIZACIÓN DE ABONOS}

Puede afirmarse que la Galicia de 1900 no conoce más que de forma limitada el uso de abonos químicos. Sin embargo, a la altura de 1919 se consumían ya un total de 171.115 quintales métricos anuales.

No debe confundirse ese reducido 0,14 ó 0,17 por 100 que corresponde al uso de los abonos químicos sobre el total de los empleados en la agricultura. Démonos cuenta, para ponderarlo debidamente, de la extraordinaria abundancia de abonos orgánicos, especialmente el estiercol de cuadra, en una agricultura tan centrada en la producción ganadera y que utiliza como sistema de estabulación la cuadra tradicional de cama de tojo para el ganado. No era fácil competir con esta oferta a tan bajo coste. Lo importante es que, para aquellos cultivos más mimados (maíz y hortícolas) o en las tierras más necesitadas, el labrador gallego de la década 1910-1920 comienza a utilizar los abonos orgánicos. Esta práctica, además, era ampliamente difundida por las organizaciones agrarias católicas a partir de 1917. En los meses de julio a septiembre de 1930, la Federación Católico-Agraria de La Coruña recibe 53 pedidos de sus sindicatos asociados por un total de 402.150 kilos de superfosfato de cal para distribuir entre sus miembros; la Federación luguesa demanda más de 200.000 y la mindoniense distribuía del mismo modo 256.785 kilos ${ }^{24}$.

${ }^{23}$ M. J. Barneiro GiL, "El Plan de Estabilización de 1959: un nuevo modelo económico para España. El papel de la agricultura en el proceso de cambio", en EQuIPo de Estudios do Agro Galego, La modernización productiva de la agricultura gallega (en prensa).

${ }^{24}$ R. VIlLARES, art. cit. 
Materias fertilizantes empleadas en la agricultura de Galicia, 1919

(Quintales métricos)

\begin{tabular}{|c|c|c|}
\hline & $Q m$ & $\%$ \\
\hline $\begin{array}{llllllllllll}\text { Estiércol de cuadra } & \ldots & \ldots & \ldots & \ldots & \ldots & \ldots & \ldots & \ldots & \ldots & \ldots \\
\text { Abonos verdes } & \ldots & \ldots & \ldots & \ldots & \ldots & \ldots & \ldots & \ldots & \ldots & \ldots & \ldots \\
\text { Basuras de poblaciones } & \ldots & \ldots & \ldots & \ldots & \ldots & \ldots & \ldots & \ldots \\
\text { Residuos de pescados } & \ldots & \ldots & \ldots & \ldots & \ldots & \ldots & \ldots & \ldots & \ldots\end{array}$ & $\begin{array}{r}105.321 .000 \\
4.998 .000 \\
634.360 \\
117.600\end{array}$ & $\begin{array}{r}94,67 \\
4,49 \\
0,57 \\
0,10\end{array}$ \\
\hline $\begin{array}{llllllllllll}\text { TOtaL } & \ldots & \ldots & \ldots & \ldots & \ldots & \ldots & \ldots & \ldots & \ldots & \ldots & \ldots\end{array}$ & 111.070 .960 & 99,83 \\
\hline 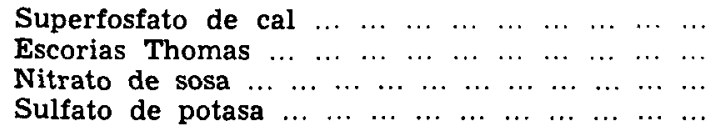 & $\begin{array}{r}98.415 \\
70.900 \\
1.200 \\
600\end{array}$ & $\begin{array}{l}0,08 \\
0,06 \\
0,00 \\
0,00\end{array}$ \\
\hline $\begin{array}{lllllllllllll}\text { TotaL } & \ldots & \ldots & \ldots & \ldots & \ldots & \ldots & \ldots & \ldots & \ldots & \ldots & \ldots\end{array}$ & 171.115 & 0,14 \\
\hline $\begin{array}{lllllllllll}\text { TOTAL } & \ldots & \ldots & \ldots & \ldots & \ldots & \ldots & \ldots & \ldots & \ldots & \ldots\end{array}$ & 111.241 .075 & 100,00 \\
\hline
\end{tabular}

Funnte: Junta Consultiva Agronómica, Materias fertilizantes empleadas en la agricultura en 1919, Madrid, 1921.

A partir de 1920-1921 comenzaron a moderarse sensiblemente los precios de los abonos químicos y, en especial, los del superfosfato de cal, lo que favorecía el aumento de su consumo. Hasta ahí el índice de precios de este producto había evolucionado siempre por encima del índice general y, por lo que hace a nuestro caso, por encima del índice de los precios de la carne de ternera de Galicia. En 1909, Valeriano Villanueva se queja enérgicamente de la «carestía de los abonos minerales más indispensables, escorias, superfosfatos y todos los compuestos del fósforo y cal que alcanzan doble precio que en el extranjero" ${ }^{25}$. Modificadas estas condiciones, el consumo de superfosfatos en 1928 asciende a 234.660 quintales métricos, a 1.690 el de nitrato sódico y a 1.470 el del sulfato de potasa, realizándose además un consumo de 14.500 quintales métricos de abonos compuestos ${ }^{26}$. Con respecto a lo que sucedía en 1919 , los consumos de estos productos aumentaron en un 138,43 por 100 en el caso de los superfosfatos, un 40,83 en el de nitrato sódico y un 145 por 100 en el caso del sulfato de potasa. En términos promedios, tan sólo diez años fueron suficientes para que el consumo de abonos químicos aumentase en un 137,30 por 100 .

${ }^{25}$ V. Viltanueva, "Organización del cultivo y la ganadería en las regiones de pequeña propiedad del Noroeste y Norte", en Semana social de España, Santiago, 1911, pp. 113-158.

${ }_{26}$ SERvicio General DE Estadistica, Anuario estadístico de España de 1928, Madrid, 1930; Junta Consultiva AGRonómica, Materias fertilizantes empleadas en la agricultura en 1919, Madr:d, 1921. 


\subsection{LAS INVERSIONES TÉCNICAS EN LA AGRICULTURA}

Durante el primer tercio del siglo xx la agricultura de Galicia tiene ante sí unas expectativas optimistas. La coyuntura económica es francamente positiva, los mercados están abiertos y relativamente asegurados y se están haciendo extraordinarios aportes técnicos a la mejora de los cultivos y a las condiciones de la cría de ganado. En el año 1921 se crea en Galicia la Misión Biológica, en la que un hombre de gran dimensión, don Cruz Gallastegui Unamuno ${ }^{27}$ hace aportes para la mejora de la agricultura de Galicia, tales como el inicio, por primera vez en Europa, de la producción de semillas híbridas del maíz en 1927; la mejora del cultivo, especies y cuidados de la patata, que significaba doblar la capacidad de producción del pais; la mejora de las plantas hortícolas y forrajeras, así como la difusión de sus semillas seleccionadas, el perfeccionamiento de la explotación del ganado de cerda, el impulso de las paradas de sementales de vacuno y la realización del control lechero-mantequero de las vacas de raza rubio gallega ${ }^{28}$.

Por otra parte, don Juan Rof Codina hacía publicar excelentes trabajos dirigidos todos ellos a la mejora de la producción ganadera de Galicia y multiplicaba sus esfuerzos divulgadores ${ }^{29}$. Eran conocidos también los trabajos sobre economía de don Luis Peña Novo ${ }^{30}$.

Los sindicatos agrarios fomentaban la introducción de maquinaria agrícola mediante los sistemas de compra y uso en común entre sus asociados ${ }^{31}$. Se celebraban abundantes exposiciones ganaderas y las ferias de nueva creación dedicaban sus primeros días a la convocatoria de concursos de ganado. Todo tipo de divulgación encontraba en este momento, además, un clima sumamente propicio para su aceptación: Galicia estaba experimentando una diná-

27 Revista dE Economía dE Galicia, núms. 25-30, tomo extraordinario dedicado a D. Cruz Gallastegui Unamuno; M. J. Barreiro GrL, "Don Cruz Gallastegui Unamuno (1891-1968). O sau labor en prol da mellora da agricultura de Galicia", en Revista Galega de Estudios Agrarios, núm. 2, 1979, pp. 269-277.

2s J. Rof Codina, "Eficacia en la economía de Galicia por la actuación de Cruz Gallastegui mediante la misión biológica", en Revista de Economía de Galicia, número cit., pp. 104-105.

${ }^{29}$ Los escritos más importantes de RoF CodrNa son: Bosquejo sanitario-zootécnico destinado al estudio de las ventajas del seguro aplicado a la ganaderia en la provincia de Lugo, Lugo, 1904; Reformas que deben implantarse en Galicia para el progreso de la agricultura, La Corun̄a, 1912; Los concursos de ganado como medio de fomentar y clarificar la ganadería, Madrid, 1915; La raza bovina gallega, Barcelona, 1916; Problemas de la ganadería española, La Coruña, 1920; Enfermedades infecto-contagiosas reinantes en la ganaderia gallega y medidas para combatirlas y evitarlas, La Coruña, 1928.

30 M. Roca Cendá, Luis Peña Novo e a súa visión da economía galega, La Coruña, 1982.

"El funcionamiento de este tipo de organizaciones lo explica J. J. Castillo, Propietarios muy pobres. Sobre la subordinación política del pequeño campesino. La Confedẹación Nacional Católico-Agraria, 1917-1942, Madrid, 1979. 
mica de asociacionismo agrario del todo inusitada en su historia moderna y contemporánea. En la mayoría de las parroquias de Galicia existía un sindicato o sociedad agraria que actuaba en el campo cooperativo, asistencial y fomentador ${ }^{32}$.

La abundancia y bajo coste del trabajo humano competía en condiciones más que favorables contra todo tipo de innovación tecnológica en la agricultura ${ }^{33}$, pero a medida que se aceleró el proceso de expulsión de mano de obra del medio agrario estos procesos de cambio se hicieron más visibles y rápidos.

\section{A modo de conclusión: la dimensión del cambio}

El proceso de cambio que se operó en la agricultura de Galicia durante los últimos años del siglo $\mathrm{xIx}$ y en el primer tercio del $\mathrm{xx}$, es de todo punto trascendental. No puede caracterizarse de otro modo una dinámica transformadora de la sociedad agraria gallega que afecta a todas las manifestaciones de la vida cotidiana: la tierra (la redención de los foros), el trabajo (la emigración y la anulación de excedentes laborales que facilitan la introducción de innovaciones tecnológicas), el capital (la ampliación de las actividades comerciales, las remesas de los propios emigrantes y el aumento de la capacidad de acumulación de las explotaciones agrarias), la organización social (el impulso del asociacionismo y sindicalismo agrario de cualquier naturaleza) y la organización de la producción (especialización productiva bajo un nuevo modelo de cálculo económico). En todo ello se evidencia la absoluta imposibilidad de que las formas económicas propias de la sociedad tradicional sigan gobernando la actividad económica, por muy amplios que sean los residuos que de las mismas sigan existiendo.

Se infravalora el cambio cuando se hace una ponderación meramente cuantitativa del mismo en aspectos como la reducida proporción del consumo de abonos químicos con respecto a los orgánicos, poniendo como referencia lo que sucede en otras latitudes geográficas, sociales y agrícolas. Sin embargo, es necesario tener en cuenta las condiciones en que aquéllos pueden o no competir ventajosamente con el estiércol de cuadra en una agricultura como la gallega y medir prudentemente hasta dónde y a partir de dónde tiene importancia decisiva su sustitución.

Sucede lo mismo cuando se afirma, por ejemplo, que a pesar del volumen de carne de vacuno que se comercializaba no tuvo una especial incidencia en

32 J. A. DURán, Agrarismo y movilización campesina en el País Gallego, 18751912, Madrid, 1977; M. J. Barreiro GII, op. cit.

${ }^{33}$ Fontani, "Transformaciones...". 
el cambio agrario, en tanto que no llegaron a constituirse explotaciones ganaderas propiamente dichas. Creo que este tipo de unidad productiva ganadera difícilmente podría llegar a constituirse en la Galicia de entonces y mientras persista vigente el modelo de agricultura familiar de tipo minifundista tan acentuado. Sin embargo, a pesar de su inexistencia no puede afirmarse que la producción ganadera de Galicia tenga una oferta débil, ni que el conjunto de cientos de miles (entre tres y cuatro) de pequeñas explotaciones mixtas no haya sido capaz de mantener en condiciones aceptables de competitividad la producción de excedentes comercializables de ganado.

Menos se puede afirmar la falta de modernización de la agricultura gallega basándose en la evidente persistencia de ese mencionado sistema agrario de naturaleza familiar y minifundista. A este respecto es necesario aceptar que la penetración del capitalismo en la agricultura ya no exige, ni exigió nunca, la anulación total del sistema de relaciones propias del mundo agrario (operación industrial de los procesos productivos, mano de obra asalariada, por ejemplo, no son condiciones indispensables para la realización de la producción agraria en el marco capitalista), sino que procede a integrar las formas campesinas en su propia racionalidad, de manera que persisten pero cumplen una distinta función social ${ }^{34}$.

En conclusión, podría afirmar que es durante el transcurso del primer tercio del siglo $\mathrm{xx}$ cuando se comienza a realizar el proceso de integración de la producción agraria de Galicia en el ámbito de cálculo del mercado capitalista, y lo hace de tal modo y con tal intensidad que, después de la obligada pausa que para la modernización productiva de la agricultura se establece entre 1936 y los últimos años de la década de 1940, el agro gallego está en condiciones de asumir, en un espacio de tiempo extraordinariamente corto (1960-1970), un proceso de transformaciones que eleva de forma espectacular el grado de mercantilización'de la actividad agraria, reduce la complementariedad de tareas productivas dentro de las explotaciones agrarias, en ese espacio de diez años elimina casi 70.000 empleos en la agricultura mientras abandonan el país 230.000 emigrantes y, por fin, entrega la mayor parte de la producción agraria al control del sistema agroindustrial.

Un cambio así, tan amplio, profundo, rápido y contundente, no podría realizarse sin raíces ni antecedentes que evitasen el caos social y económico.

\footnotetext{
34 Equipo de Estudios do Agro Galego, op. cit.; E. Ptrez Touriño, "Dominación do capitalismo monopolista e cámbeos na economía galega", en Materiáis, núm. 5, 1977; P. Colıvo, "O proceso de mercantilización da agricultura galega", en Nova Galicia, núms. 1-2, 1978; E. Pérez Touriño, Agricultura y capitalismo. Análisis de la pequeña producción campesina, Madrid, 1983.
} 